\title{
ANWENDUNGEN DER WERTVERTEILUNGSLEHRE AUF GEWÖHNLICHE DIFFERENTIALGLEICHUNGEN
}

\section{H. WITTICH}

Die Entwicklung der Theorie der gewöhnlichen Differentialgleichungen im Komplexen zeigt, wie Fortschritte in der Funktionentheorie zu neuen Ergebnissen und Fragestellungen in der Theorie der Differentialgleichungen führten. Das gilt auch für die Theorie der Wertverteilung. Die erste Anwendung der Nevanlinnaschen Theorie auf gewöhnliche Differentialgleichungen im Komplexen gab 1932 K. Yosida (K. Yosida [1]); er bewies den bekannten Satz von Malmquist (Acta Math. 36 (1913)) mit Hilfsmitteln der Wertverteilungslehre und gab Verallgemeinerungen an. Seit 1950 entstanden zahlreiche Untersuchungen zum Thema ,Wertverteilung und gewöhnliche Differentialgleichungen im Komplexen“. Zum gleichen Thema sollen in diesem Vortrag einige Bemerkungen gemacht werden.

1. In $w^{\prime}=P(z, w)=a_{0}(z) w^{n}+\ldots+a_{0}(z)$ sei $P$ ein Polynom in $z$ und $w$ und $w(z)$ eine in $|z|<\infty$ eindeutige, nichtrationale Lösung: $w^{\prime}(z) \equiv P(z, w(z))$. Für die Charakteristik gilt $T(r, P(z, w(z)))=T\left(r, w^{\prime}\right)=n \cdot T(r, w)+O(\log r)$ und mit

$$
\begin{gathered}
T\left(r, w^{\prime}\right) \leqq T(r, w)+\bar{N}(r, w)+S(r), \\
n \cdot T(r, w) \leqq T(r, w)+\bar{N}(r, w)+S(r) \leqq 2 T(r, w)+S(r) .
\end{gathered}
$$

Es folgt: Ganze transzendente Lösungen sind nur für $n=1$ möglich, meromorphe Lösungen nur für $n=2$. Jede Lösung von $w^{\prime}=a_{0}(z)+a_{1}(z) w$ ist eine ganze Funktion. Für $n=2$ liegt eine Riccatische Differentialgleichung vor. Allgemeiner gilt der Satz von Malmquist:

Jede im Großen eindeutige Lösung von $w^{\prime}=R(z, w)$ (rational in $z$ und $w$ ) ist entweder eine rationale Funktion oder es liegt eine Riccatische Differentialgleichung $w^{\prime}=a_{0}(z)+a_{1}(z) w+a_{2}(z) w^{2}$ vor. Im Jahre 1932 gab K. Yosida einen Beweis dieses Satzes mit Hilfsmitteln der Wertverteilungslehre. Wie oben folgt aus der Differentialgleichung, daß die rationale Funktion $R(z, w) \operatorname{vom}$ Grad $\leqq 2$ (in $w$ ) sein muß. Da das auch für jede Funktion $y=1 /(w-\alpha)$ bei beliebigem $\alpha$ gelten muß, folgt $R(z, w)=a_{0} w^{2}+a_{1} w+a_{2}$ mit in $z$ rationalen Koeffizienten $a_{0}(z), \ldots, a_{2}(z)$, also die Behauptung. 
Die Differentialgleichung $w^{\prime}=P(z, w) / Q(z, w)=R(z, w)$ läßt sich auf die Form bringen

$$
w^{\prime}=w^{2}+a_{1}(z) w+a_{0}(z)+\frac{P_{h}(z, w)}{Q(z, w)}, \quad h \leqq q-1 ;
$$

$h$ bzw. $q$ ist der Grad von $w$ in $P_{h}$ bzw. $Q$.

$$
F(z)=\frac{P_{h}(z, w(z))}{Q(z, w(z))}=w^{\prime}(z)-w^{2}(z)-a_{1}(z) w(z)-a_{0}(z)
$$

ist in $R<|z|<\infty$ holomorph und hat unendlich viele Nullstellen (in den unendlich vielen Polstellen von $w(z))$. Ist $w(z)$ von endlicher Ordnung und $m(r, w)=O(\log r)$, dann folgt $m(r, F)=m\left(r, w^{\prime}-w^{2}-a_{1} w-a_{0}\right)=T(r, F)=O(\log r)$. Das wiederspricht der Folgerung, daß $F(z)$ unendlich viele Nullstellen hat: $Q(z, w)$ muß also unabhängig von $w$ sein.

Mit einer passenden natürlichen Zahl $g$ gilt für alle hinreichend großen $|z|$, die $|w(z)|>|z|^{g} \quad$ erfüllen, $\quad w^{\prime}(z) / w^{2}(z)=1+h(z), \quad|h(z)| \leqq 1 / 2$. Diese Darstellung erlaubt die Konstruktion von punktfremden Kreisscheiben $U(\zeta)$ um die einfachen Polstellen mit den Eigenschaften: In $U(\zeta)$ gilt $(2 / 3)(1 /|z-\zeta|) \leqq|w(z)| \leqq 2 /|z-\zeta|$ und $|w(z)| \leqq C|z|^{k}$ außerhalb $U(\zeta)$ und $|z| \geqq R$. Daraus folgert man $m(r, w)=O(\log r)$ und $N(r, w) \leqq C r^{k+1}$, also die Behauptungen: $w(z)$ ist von endlicher Ordnung und $m(r, w)=O(\log r)$.

$$
w^{\prime}=a(z)+b(z) w+c(z) w^{2}
$$

die Koeffizienten rationale Funktionen, dann ist die Existenz von in $|z|<\infty$ eindeutigen Lösungen nicht gesichert, während bei Polynomkoeffizienten jede Lösung von (1) in $|z|<\infty$ meromorph ist. Mit der Formel von Ahlfors-Shimizu folgt aus (1) die schon bekannte Tatsache, daß die Ordnung $\lambda(w)$ endlich ist. Genauer gilt $\lambda(w)=1+d / 2 \geqq 1$, wobei sich $d$ aus der Entwicklung von $B(z)=a c-b^{2} / 4+$ $b^{\prime} / 2-(b / 2)\left(c^{\prime} / c\right)=B_{0} z^{d}(1+O(1 / z))$ bestimmt. Für rationale Koeffizienten ist $d=-1$ möglich; dann ist $\lambda(w) \geqq 1 / 2$. Es gilt also: Jede in $|z|<\infty$ eindeutige analytische Funktion der Ordnung $<1 / 2$, die $w^{\prime}=R(z, w), R(z, w)$ rational in $z$ und $w$, genügt, ist eine rationale Funktion (Wittich [1], [4]).

Für (1) schreiben wir

$$
\begin{gathered}
w^{\prime}=D(z)+b(z)(w-k)+c(z)\left(w^{2}-k^{2}\right), \\
D(z)=a(z)+k b(z)+k^{2} c(z),
\end{gathered}
$$

$k$ eine beliebige komplexe Zahl. Bei $D(z) \not \equiv 0$ folgt daraus $m(r, 1 /(w-k))=O(\log r)$. Für jeden endlichen Wert $k$ und $\infty$ gilt daher

$$
\lim _{r \rightarrow \infty} \frac{N(r, 1 /(w-k))}{T(r, w)}=1, \quad \lim _{r \rightarrow \infty} \frac{N(r, w)}{T(r, w)}=1 .
$$


Nur für höchstens zwei Werte $k_{1}, k_{2}$ ist $D(z) \equiv 0$ möglich. Man erhält dann die Differentialgleichungen

$$
\begin{aligned}
& w^{\prime}=c(z)\left(w-k_{1}\right)\left(w-k_{2}\right) \\
& w^{\prime}=(w-k)(b(z)+c(z) k+c(z) w) \\
& w^{\prime}=c(z)(w-k)^{2} .
\end{aligned}
$$

Jede transzendente Lösung der ersten Differentialgleichung hat die Picardschen Ausnahmewerte (abgekürzt P. A. W.) $k_{1}$ und $k_{2}$. Für die transzendenten Lösungen der zweiten Differentialgleichung ist $k$ P. A.W. Als genaue Defektrelation gilt $\Phi_{e}+$ $\delta\left(k_{1}\right)+\delta\left(k_{2}\right)=2, \Phi_{e}+\delta(k)=2$. Der Verzweigungsindex für jeden Wert $k$ einschließlich $\infty$ ist stets Null. Faktorisierbare Lösungen von (1) mit Polynomkoeffizienten sind von E. Mues [1] untersucht worden.

Aus $w^{\prime \prime}=P(z, w)=a_{0}(z) w^{n}+\ldots$ folgt $n T(r, w) \leqq T(r, w)+2 \bar{N}(r, w)+S(r)$, also: In $|z|<\infty$ eindeutige analytische Lösungen sind nur für $n \leqq 3$ möglich. Es gibt solche Differentialgleichungen, nämlich die beiden ersten Differentialgleichungen von Painlevé

$$
w^{\prime \prime}=6 w^{2}+A_{0} z+B \quad \text { und } \quad w^{\prime \prime}=2 w^{3}+\left(B_{0} z+B\right) w+C .
$$

Jede Lösung ist eine in $|z|<\infty$ meromorphe Funktion.

Für $A_{0}=B_{0}=0$ lassen sich diese Differentialgleichungen mittels elliptischer Funktionen lösen. Diese Lösungen haben mindestens einen vollständig verzweigten endlichen Wert. Diese Tatsache ist charakteristisch, denn es gilt der Satz: Die beiden ersten Painlevéschen Differentialgleichungen haben genau dann Lösungen mit mindestens einem vollständig verzweigten endlichen Wert, wenn $A_{0}$ und $B_{0}=0$ sind (Schubart-Wittich [1]).

2. Auf weitere Verallgemeinerungen des Satzes von Malmquist durch K. Yosida, I. Laine, Sh. Strelitz u. a. gehe ich nicht weiter ein, da diese Resultate in einem Satz von N. Steinmetz (Karlsruher Dissertation 1978) enthalten sind. Betrachtet werden Differentialgleichungen der Form

$$
P\left(z, w, w^{\prime}, \ldots, w^{(n)}\right)=\frac{A(z, w)}{B(z, w)}=H(z, w) ;
$$

$A(z, w)$ und $B(z, w)$ sind ganz in $z$ und $w$. Die linke Seite ist eine endliche Summe von Ausdrücken der Form $a(z) w^{j_{0}}\left(w^{\prime}\right)^{j_{1}} \ldots\left(w^{(n)}\right)^{j_{n}}$ mit in $|z|<\infty$ holomorphen Koeffizienten. Es sei $d=\max \left(j_{0}+2 j_{1}+\ldots+(n+1) j_{n}\right)$. Eine in $|z|<\infty$ meromorphe Funktion $f(z)$ heißt zulässig (bezüglich der betrachteten Differentialgleichungen), wenn 1) alle Koeffizienten $a(z)$ der Bedingung $T(r, a)=O(\log r, T(r, f))=S(r, f)$ genügen und 2) $T(r, H(z, \tau))=S(r, f)$ gilt für alle $\tau \in M \subset C$, wobei die Menge $M$ einen endlichen Häufungspunkt hat. Jede in $|z|<\infty$ meromorphe Funktion $f(z)$ ist für die Differentialgleichung $w^{\prime}=R(z, w)$ zulässig. Der Satz lautet: 
Besitzt die Differentialgleichung (3) eine in $|z|<\infty$ zulässige Lösung, dann ist $H(z, w)$ ein Polynom in w vom Grad $\leqq d$.

Wichtiges Beweismittel ist der zweite Hauptsatz.

In der Differentialgleichung $w^{(n)}+a_{n-1}(z) w^{(n-1)}+\ldots+a_{0}(z) w=f(z, w), a_{j}(z)$ Polynome, sei $f(z, w)$ ein Polynom in $z$ und eine in $w$ ganze, nichtlineare Funktion. Für diese Differentialgleichung ist jede ganze transzendente Funktion zulässig.

Die Differentialgleichung läßt aber keine ganze transzendente Lösung zu; $w(z)$ sei eine solche Lösung. Dann ist $f(z, w)$ ein Polynom vom Grad $g>1$ in $w$. Es folgt

$$
\begin{gathered}
T(r, f(z, w(z)))=m(r, f(z, w(z))) \\
=g \cdot T(r, w)+O(\log r)=m\left(r, w^{(n)}+\ldots+a_{0}(z) w\right) \leqq m(r, w)+S(r),
\end{gathered}
$$

also $g T(r, w) \leqq T(r, w)+S(r)$. Das ist ein Widerspruch. Für die binomische Gleichung $\left(w^{\prime}\right)^{n}=R(z, w)$ ist jede in $|z|<\infty$ eindeutige nichtrationale analytische Funktion $f(z)$ zulässig. Für eine zulässige Lösung gilt: $R(z, w)=a_{0}(z) w^{k}+\ldots+a_{k}(z)$, $a_{j}(z)$ rationale Funktionen und $n \leqq k \leqq 2 n$; für $n=k$ hat $w(z)$ höchstens endlich viele Pole.

3. In $|z|<\infty$ eindeutige Lösungen algebraischer Differentialgleichungen erster Ordnung $F\left(z, w, w^{\prime}\right)=0$ sind von endlicher Ordnung. Genauer gilt die Aussage:

$w(z)$ ganz transzendent: $\frac{1}{2} \leqq \lambda(w)<\infty ; w(z)$ meromorph: $0 \leqq \lambda(w)<\infty$.

Nach Bank-Kaufman [1] gibt es Differentialgleichungen $w^{\prime n}=a(z) P(w)$, die meromorphe Lösungen der Ordnung Null haben. Algebraische Differentialgleichungen der Ordnung $n \geqq 2$ können Lösungen von unendlich hoher Ordnung haben, wie $w w^{\prime \prime}-w^{\prime 2}-w w^{\prime}=0$ mit der Lösung $w(z)=\exp \left(e^{z}\right)$ zeigt. Sind in der Differentialgleichung

$$
F\left(z, w, w^{\prime}\right)=\sum_{j, k \geqq 0} a_{j k}(z) w^{j} w^{\prime k}=0
$$

die Koeffizienten ganze Funktionen, dann sind, wie $w^{\prime}=e^{z} w$ mit $w(z)=\exp \left(e^{z}\right)$ zeigt, ganze Lösungen der Ordnung $\infty$ möglich. Nach S. Bank [1] gilt bei $\lambda\left(a_{j k}(z)\right)<\infty$ für jede reelle Zahl $\lambda>\lambda\left(a_{j k}\right)$ die Abschätzung

$$
\log M(r, w) \leqq \exp \left(r^{\lambda}\right), \quad r>r_{0}(\lambda),
$$

$w(z)$ eine ganze Lösung von (4).

Es gibt Differentialgleichungen (4) mit meromorphen Lösungen $w(z)$, deren Charakteristiken $T(r, w)$ nicht durch die Koeffizienten $a_{j k}(z)$ allein abgeschätzt werden können (wie bei ganzen transzendenten Lösungen). Für die Charakteristik $T(r, w)$ einer meromorphen Lösung $w(z)$ von (4) gilt

$$
T(r, w)<\exp \left(r^{\lambda}\right)+K \cdot r N(a r, w), \quad a>1 .
$$


Weitere Resultate für Differentialgleichungen höherer Ordnung verdankt man S. Bank und Mitarbeitern (vgl. S. Bank-Laine [1]).

4. In

$$
L(w)=w^{(n)}+a_{n-1}(z) w^{(n-1)}+\ldots+a_{0}(z) w=0, \quad a_{0}(z) \not \equiv 0,
$$

seien die Koeffizienten $a_{j}(z)$ ganz. Dann sind alle Lösungen $w(z)$ ganze Funktionen. Übergang zu einem äquivalenten System und Herleitung einer Differentialungleichung für $y(r)=\left(\sum_{j=1}^{n}\left|w_{j}\right|^{2}\right)^{1 / 2}$ zeigt, daß $M(r, w)$ durch die Maximalbeträge der Koeffizienten nach oben abgeschätzt werden kann. Sind die $a_{j}(z)$ Polynome, dann gilt $\lambda(w)<\infty$ für alle Lösungen von (D). Umgekehrt sei $\lambda(w)<\infty$. Für $n=2$ ergibt sich wegen $a_{1}(z)=-w^{\prime}(z) / w(z) \quad m\left(r, a_{1}\right)=O(\log r)$ und wegen $a_{0}(z)=-w^{\prime \prime} / w-$ $a_{1}(z)\left(w^{\prime} / w\right) \quad m\left(r, a_{0}\right)=O(\log r): a_{1}(z)$ und $a_{0}(z)$ sind Polynome. Entsprechende Betrachtungen, kombiniert mit der Reduktionsmethode von L. Fuchs, liefern für beliebiges $n$ : Die Koeffizienten in (D) sind genau dann Polynome, wenn jede Lösung von endlicher Ordnung ist.

Für manche Untersuchungen ist es zweckmäßig, gewisse Fundamentalsysteme von (D) auszuzeichen. Sie haben die Eigenschaften: Die Ordnungen sind $0 \leqq \lambda_{1}<$ $\lambda_{2}<\ldots<\lambda_{v}, 1 \leqq v \leqq n$. Zu $\lambda_{j}$ gehören $\mu_{j}$ linear unabhängige Lösungen $v_{1}, v_{2}, \ldots, v_{\mu_{j}}$, $\mu_{1}+\mu_{2}+\ldots+\mu_{v}=n$. Jede Linearkombination $c_{1} v_{1}+\ldots+c_{\mu_{j}} v_{\mu_{j}} \neq 0$ hat die Ordnung $\lambda_{j}$. Diesem ausgezeichneten Fundamentalsystem entspricht eine Aufteilung des Lösungsraumes $V$ von $L(w)=0$

$$
V=V_{1} \oplus V_{2} \oplus \ldots \oplus V_{v}
$$

$V_{j}$ ist ein Unterraum der Dimension $\mu_{j}$. Jedes $w \in V_{j}, w(z) \not \equiv 0$, hat die Ordnung $\lambda_{j}$. Bei Polynomkoeffizienten $a_{j}(z)$ vom Grad $\alpha_{j}$ sind weitergehende Aussagen über die $\lambda_{j}$ möglich. Für $j=0,1, \ldots, n$ bildet man $g=\max _{j=0}^{n-1}\left(\alpha_{j}+n-j\right), \quad \beta_{j}=g-$ $\left(\alpha_{j}+n-j\right), Q_{j}=\left(j, \beta_{n-j}\right), \alpha_{n}=0$. Der kürzeste Streckenzug, der die Punkte $Q_{j}$ von unten konvex umschließt, heißt das Diagram $\mathfrak{D}$ von (D). Dem absteigenden Teil entnimmt man die nichtnegativen rationalen Zahlen $\lambda^{\prime}=v / u$. Unter diesen $\lambda^{\prime}$ kommen die $\lambda_{j}$ vor, wobei $\mu_{j} \leqq v$ gilt. Insbesondere ist $\lambda_{v}=\max \lambda^{\prime}$. Die zur Herleitung

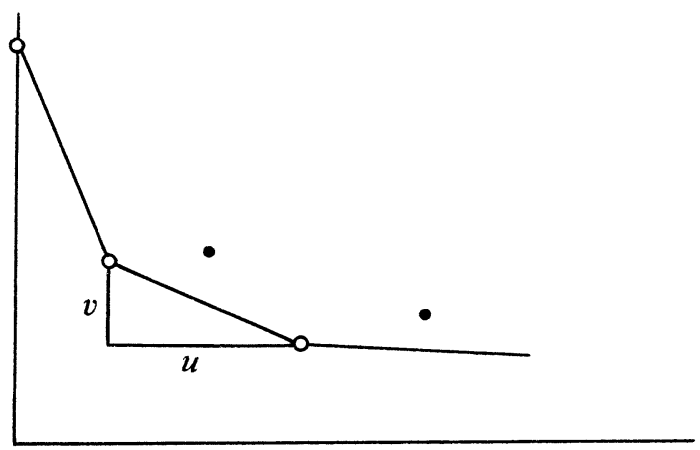


von $\mathfrak{D}$ benutzten Methoden (Methode des Zentralindex) liefern: Die Lösungen $w(z)$ sind von regulärem Wachstum der Ordnung $\lambda_{j}$. Die Gleichung $\lambda(w)=0$ gilt genau dann, wenn $w(z)$ ein Polynom ist. Mindestens eine Lösung ist ganz transzendent; (D) hat also höchstens $n-1$ linear unabhängige Polynomlösungen. Weiter ist $\lambda_{\nu} \geqq 1$ und $=1$ genau dann, wenn (D) konstante Koeffizienten hat.

$\mathfrak{M}$ sei die Menge aller ganzen Funktionen, die Lösungen von Differentialgleichungen (D) mit Polynomkoeffizienten und $n \in N$ sind. $\mathfrak{M}$ ist ein linearer Raum, der die weitere Eigenschaft hat, daß mit $w_{1}, w_{2} \in \mathfrak{M}$ auch $w_{1} \cdot w_{2} \in \mathfrak{M}$ ist (FrankWittich [1]).

Für (D) mit Polynomkoeffizienten sei $\omega=\left(w_{1}, \ldots, w_{n}\right)$ ein beliebiges Fundamentalsystem und $S(\omega)=\sum_{j=1}^{n} \lambda\left(w_{j}\right)$. Genau die ausgezeichneten Fundamentalsysteme machen $S(\omega)$ zum Minimum $m(L)$; man spricht daher auch von Minimalsystemen. Für alle Differentialgleichungen $L(w)=0$ ohne Polynomlösungen gilt $m(L) \geqq n$ und $=n$ genau dann, wenn $L(w)=0$ konstante Koeffizienten hat. $\mathrm{Ob}$ diese Aussage ohne Zusatz $\lambda_{1}>0$ richtig ist, scheint nicht bekannt zu sein.

Die Gleichung $\lambda_{v}=\infty$ ist nur möglich, wenn mindestens einer der Koeffizienten $a_{j}(z)$ ganz transzendent ist. Der Koeffizient $a_{0}(z)$ sei ganz transzendent, die restlichen Koeffizienten $a_{1}(z), \ldots, a_{n-1}(z)$ seien Polynome. Dann gilt für jede Lösung $w(z) \not \equiv 0 \lambda(w)=\infty$. Es sei für eine Lösung $w_{0}(z) \lambda\left(w_{0}\right)<\infty$. Dann folgt aus $a_{0}(z)=-w_{0}^{n}(z) / w_{0}(z)-\ldots-a_{1}(z) w_{0}^{\prime} / w_{0} m\left(r, a_{0}\right)=O(\log r)$. Die Ungleichung $\lambda\left(w_{0}\right)<\infty$ führt zum Widerspruch. Analoge Überlegungen führen zum Resultat:

Ist unter den Koeffizienten $a_{0}(z), \ldots, a_{n-1}(z) a_{k}(z)$ der letzte transzendente Koeffizient (also $a_{k+1}(z), \ldots, a_{n-1}(z)$ Polynome) dann ist $\mu_{\nu} \geqq n-k$. In einem ausgezeichneten Fundamentalsystem kommen mindestens $n-k$ linear unabhängige Lösungen der Ordnung $\infty$ vor (M. Frei [1]).

Beim Umkehrproblem wird die Aufgabe behandelt, zu gegebenen $\lambda_{j}, \mu_{j}$ Differentialgleichungen (D) zu konstruieren, deren Minimalsysteme die Größen $\lambda_{j}, \mu_{j}$ aufweisen.

$\lambda_{v}=\infty$ : Gegeben sind die reellen Zahlen $\lambda_{1}, \ldots, \lambda_{v}$ mit $0 \leqq \lambda_{1}<\lambda_{2}<\ldots<\lambda_{v}=\infty$ und die natürlichen Zahlen $\mu_{1}, \ldots, \mu_{v}$ mit $\mu_{1}+\mu_{2}+\ldots+\mu_{v}=n$. Man konstruiere eine Differentialgleichung (D) mit einem Minimalsystem

$$
\begin{aligned}
& 0 \leqq \lambda_{1}<\lambda_{2}<\ldots<\lambda_{v} \\
& \mu_{1}+\mu_{2}+\ldots+\mu_{v}=n .
\end{aligned}
$$

Diese Aufgabe ist, wie eine Konstruktion von J. Nikolaus [1] zeigt, lösbar. Die Gleichung $\lambda(w)=0$ ist auch für ganze transzendente Lösungen $w(z)$ möglich.

$\lambda_{v}<\infty$ : Gegeben sind die rationalen Zahlen $0 \leqq \lambda_{1}<\lambda_{2}<\ldots<\lambda_{v}<\infty$ und die natürlichen Zahlen $\mu_{1}, \ldots, \mu_{v}$ mit $\mu_{1}+\ldots+\mu_{v}=n$. Gibt es eine Differentialgleichung (D) (mit Polynomkoeffizienten) mit minimalen Fundamentalsystemen, in denen die Ordnungen $\lambda_{j}$ mit den Vielfachheiten $\mu_{j}$ auftreten? 
In dieser allgemeinen Fassung ist die Aufgabe nicht lösbar. Für $n=3 \quad \lambda_{1}=1 / 2$, $\lambda_{2}=3 / 4$ mit $\mu_{1}=2, \mu_{2}=1$ gibt es keine Lösung. Sonst müßte wegen $\lambda_{1}>0 \mu_{1} \lambda_{1}+$ $\mu_{2} \lambda_{2} \geqq 3$ sein, was nicht der Fall ist. Für $n=3 \lambda_{1}=1 / 2, \mu_{1}=1, \lambda_{2}=2, \mu_{2}=2$ ist das Umkehrproblem lösbar. Lösungen sind alle Differentialgleichungen $w^{\prime \prime \prime}+a_{2}(z) w^{\prime \prime}+$ $a_{1}(z) w^{\prime}+a_{0}(z) w=0$ mit $a_{0}(z) \equiv A_{0} \neq 0, a_{1}(z) \equiv A_{1} \neq 0, a_{2}(z)=A z+B, A \neq 0$.

In den folgenden Fällen ist Lösbarkeit gesichert:

a) Die $\lambda_{j}$ sind natürliche Zahlen.

b) Für die nicht negativen ganzen Zahlen $\lambda_{j}$ gilt $0 \leqq \lambda_{1}<\lambda_{2}<\ldots<\lambda_{v}$ und $\lambda_{v} \geqq n$ (Frank-Nikolaus [1]).

Hat (D) Polynomkoeffizienten, dann folgt für $c \neq 0, \infty$ aus

$$
w^{(n)}+\ldots+a_{0}(z)(w-c)+c a_{0}(z) \equiv 0
$$

$m(r, 1 /(w-c))=O(\log r)$, also $\delta(w, c)=0$ für jede Lösung $w(z)$.

Eine Lösung von (D) kann höchstens Null als endlichen defekten Wert haben. Weiter gilt $\lim _{r \rightarrow \infty}(N(r, a) / N(r, b))=1, a \cdot b \neq 0$. Aus (D) folgt weiter $m\left(r, w / w^{\prime}\right)=$ $O(\log r)$. Zusammen mit $m\left(r, w^{\prime} / w\right)=O(\log r)$ erhält man $m\left(r, w^{\prime}\right)=m(r, w)+$ $O(\log r), m\left(r, 1 / w^{\prime}\right)=m(r, 1 / w)+O(\log r)$, also

$$
N\left(r, \frac{1}{w^{\prime}}\right)+m\left(r, \frac{1}{w}\right)=T(r, w)+O(\log r)=T\left(r, w^{\prime}\right)+O(\log r) .
$$

5. Wir betrachten noch kurz lineare Differentialgleichungen zweiter Ordnung, deren Koeffizienten $a_{j}(z)$ isolierte Singularitäten haben können. Ist $\zeta$ eine solche Stelle, dann sind die $a_{j}(z)$ in $0<|z-\zeta|<R$ holomorph. Dort existieren kanonische Fundamentalsysteme, die jetzt betrachtet werden. Wir setzen $\zeta=\infty$. Die Differentialgleichungen seien von der Form

$$
z^{2} w^{\prime \prime}+z a(z) w^{\prime}+b(z) w=0 ;
$$

$a(z), b(z)$ sind in $G=\{z ; R<|z|<\infty\}$ holomorph. Ein kanonisches Fundamentalsystem bei $z=\infty$ ist von der Form

$$
\begin{array}{lll}
w_{1}(z)=z^{\varrho_{1}} u(z) & w_{1}(z)=z^{\varrho} u(z) \\
w_{2}(z)=z^{\varrho_{2}} v(z) & \text { und bei } \quad \varrho_{1}=\varrho_{2}=\varrho & \\
& & w_{2}(z)=z^{\varrho}(v(z)+c \cdot u(z) \log z) .
\end{array}
$$

Die in $G$ holomorphen Funktionen $u(z), v(z)$ verhalten sich genau dann rational in $z=\infty$, wenn $\lim _{z \rightarrow \infty} a(z) / z=\lim _{z \rightarrow \infty} b(z)=0$ gilt $(z=\infty$ ist eine Stelle der Bestimmtheit). Nach den Resultaten bei Differentialgleichungen mit ganzen Koeffizienten wird man erwarten, daß sich $a(z)$ und $b(z)$ genau dann rational in $z=\infty$ verhalten, wenn $\lambda(u, \infty)$ und $\lambda(v, \infty)$ (d. s. lokale Ordnungen für $z=\infty$ ) endlich sind: $L(\infty)=$ $\lambda(u, \infty)+\lambda(v, \infty)<\infty$. Das ist tatsächlich der Fall, auch für $n>2$. Verhalten sich $a(z), b(z)$ rational in $z=\infty$

$$
a(z)=A z^{\alpha}\left(1+O\left(\frac{1}{z}\right)\right), \quad b(z)=B z^{\beta}\left(1+O\left(\frac{1}{z}\right)\right),
$$


dann berechnet sich $L(\infty)$ aus $\alpha$ und $\beta$. Der Punkt $z=\infty$ ist genau dann Stelle der Bestimmtheit, wenn $L(\infty)=0$ gilt. Für die Unbestimmtheitsstelle $z=\infty$ ist $L(\infty)$ eine natürliche Zahl.

Gegeben seien jetzt $v$ Punkte $z_{1}, z_{2}, \ldots, z_{v}=\infty . G_{v}$ ist dann die in diesen Punkten punktierte Vollebene. In $G_{v}$ seien $a(z), b(z)$ holomorph. Zu jeder Stelle $z_{j}$ gehören kanonische Fundamentalsysteme mit den eindeutigen Funktionen $u_{j}(z), v_{j}(z)$ und $L\left(z_{j}\right)=\lambda\left(u_{j}, z_{j}\right)+\lambda\left(v_{j}, z_{j}\right) . \quad L\left(z_{j}\right)<\infty$ gilt genau dann, wenn sich $a(z), b(z)$ rational in $z_{j}$ verhalten. Danach sind die Koeffizienten $a(z), b(z)$ genau dann rationale Funktionen, wenn alle $L\left(z_{j}\right)$ endlich sind. $L\left(z_{j}\right)=0$ kennzeichnet die Differentialgleichungen vom Fuchs'schen Typus.

Für $\quad v=2$ sei $z_{1}=0, z_{2}=\infty . \quad L(0)=0, L(\infty)=0$ führt zur Eulerschen Differentialgleichung. Aus den Forderungen $L(0)=0, L(\infty)=1$ folgt zunächst wegen $L(\infty)=1$

$$
z^{2} w^{\prime \prime}+z\left(a_{0}+\frac{a_{-1}}{z}+\ldots\right) w^{\prime}+\left(b_{1} z+b_{0}+\frac{b_{-1}}{z}+\ldots\right) w=0, \quad b_{1} \neq 0 .
$$

Da $z=0$ Stelle der Bestimmtheit sein soll, folgt weiter $a_{-1}=\ldots=b_{-1}=\ldots=0$

$$
z^{2} w^{\prime \prime}+z a_{0} w^{\prime}+\left(b_{1} z+b_{0}\right) w=0 .
$$

Die determinierende Gleichung bei $z=0$ lautet $\varrho^{2}-\left(1-a_{0}\right) \varrho+b_{0}=0$. Mit $a_{0}=1$ gilt für die Wurzeln $\varrho_{1}, \varrho_{2} \varrho_{1}+\varrho_{2}=0$. Durch eine passende Transformation $z \rightarrow c z$ erhalten wir schließlich die Differentialgleichung

$$
z^{2} w^{\prime \prime}+z w^{\prime}-\left(z+k^{2}\right) w=0,
$$

die durch Zylinderfunktionen gelöst wird. Zur konfluenten hypergeometrischen Differentialgleichungen führt $L(0)=0, L(\infty)=2$. Die Forderung $L(0)=L(\infty)=1 \mathrm{ergibt}$ nach der Transformation $z=e^{2 i t}$ Mathieusche Differentialgleichungen.

K. Böhmer [1] hat diesen Ansatz (Wittich [3]) weiter entwickelt und die Gleichungen der mathematischen Physik mit rationalen Koeffizienten einheitlich durch Wachstums- und Symmetriebedingungen gekennzeichnet.

\section{Literatur}

BANK, S. [1]: A note on algebraic differential equations whose coefficients are entire functions of finite order. - Ann. Scoula Norm. Sup. Pisa Sci. Fis. Mat. 26, 1972, 291-297.

BANK, S. B., and R. P. KaufMan [1]: On meromorphic solutions of first-order differential equations. - Comment. Math. Helv. 51, 1976, 289-299.

BANK, S., and I. LAINE [1]: On the growth of meromorphic solutions of linear and algebraic differential equations. - Math. Scand. 40, 1977, 119-126.

BöHMER, K. [1]: Charakterisierung von linearen Differentialgleichungen durch Wachstumsbedingungen I u. II. - Arch. Math. (Basel) 26, 1975, 64-74 u. 170-177.

Frank, G., und J. Nikolaus [1]: Zum Umkehrproblem für lineare Differentialgleichungen mit Polynomen als Koeffizienten. - Math. Ann. 189, 1970, 169-177. 
Frank, G., und H. WitTich [1]: Zur Theorie linearer Differentialgleichungen im Komplexen. Math. Z. 130, 1973, 363-370.

FrEI, M. [1]: Über die Lösungen linearer Differentialgleichungen mit ganzen Funktionen als Koeffizienten. - Comment. Math. Helv. 35, 1961, 201-222.

MuES, E. [1]: Über faktorisierbare Lösungen von Riccatischen Differentialgleichungen. - Math. Z. 121, 1971, 145-156.

Nikolaus, J. [1]: Konstruktion linearer Differentialgleichungen mit gegebener Struktur des Lösungsraumes. - Ann. Acad. Sci. Fenn. Ser. A I Math. 451, 1969, 1-14.

Schubart H., und H. Wittich [1]: Über die Lösungen der beiden ersten Painlevéschen Differentialgleichungen. - Math. Z. 66, 1957, 364-370.

Wiтtich, H. [1]: Zur Theorie der Riccatischen Differentialgleichung. - Math. Ann. 127, 1954, $433-440$.

Wiтtich, H. [2]: Zur Kennzeichnung linearer Differentialgleichungen mit konstanten Koeffizienten. - Festband zum 70. Geburtstag von Rolf Nevanlinna. Vorträge, gehalten anläßlich des Zweiten Rolf Nevanlinna-Kolloquiums in Zürich vom 4-6. November 1965. Springer-Verlag, Berlin-Heidelberg-New York 1966, 128-134.

Wittich, H. [3]: Zur Theorie linearer Differentialgleichungen im Komplexen. - Ann. Acad. Sci. Fenn. Ser. A I Math. 379, 1966, 1-18.

Wiтtich, H. [4]: Neuere Untersuchungen über eindeutige analytische Funktionen. - Ergebnisse der Mathematik und ihrer Grenzgebiete 8, zweite Aufl., Springer-Verlag, Berlin-Heidelberg-New York, 1968.

YosidA, K. [1]: A generalisation of a Malmquist's theorem. - Jap. J. Math. 9, 1933, 253-256.

Hansjakobstrasse 14

7500 Karlsruhe

Deutschland

Eingegangen am 14. Oktober 1981 